LAWRENCE LIVERMORE NAT IO N A L LABORATORY

\title{
The Next Generation Microlensing Search: SuperMacho
}

A.J. Drake, A.C. Becker, A. Clocchiati, K.H. Cook, R. Covarrubias, R. Hiriart, S. Keller, G. Miknaitis, S. Nilolaev, K. Olsen, G. Prochter, A. Rest, B.P. Schmidt, C. Smith, C.W. Stubbs, N. Suntzeff, D.L. Welch

October 27, 2003

the International Conference DARK 2002 Cape Town, South Africa February 4, 2002 through February 9, 2002 
This document was prepared as an account of work sponsored by an agency of the United States Government. Neither the United States Government nor the University of California nor any of their employees, makes any warranty, express or implied, or assumes any legal liability or responsibility for the accuracy, completeness, or usefulness of any information, apparatus, product, or process disclosed, or represents that its use would not infringe privately owned rights. Reference herein to any specific commercial product, process, or service by trade name, trademark, manufacturer, or otherwise, does not necessarily constitute or imply its endorsement, recommendation, or favoring by the United States Government or the University of California. The views and opinions of authors expressed herein do not necessarily state or reflect those of the United States Government or the University of California, and shall not be used for advertising or product endorsement purposes. 


\title{
The Next Generation Microlensing Search: SuperMacho.
}

\author{
A.J. Drake ${ }^{1}$, A.C. Becker ${ }^{2}$, A. Clocchiati ${ }^{3}$, K.H. Cook $^{1}$, R. Covarrubias ${ }^{4}$, R. \\ Hiriart $^{5}$, S. Keller ${ }^{1}$, G. Miknaitis ${ }^{4}$, S. Nikolaev ${ }^{1}$, K. Olsen ${ }^{5}$, G. Prochter ${ }^{1}$, A.

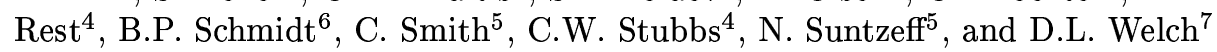 \\ 1 IGPP, Lawrence Livermore Nat. Lab., Livermore, CA 94550, USA \\ 2 Bell Labs, Lucent Technol., 600 Mountain Avenue, Murray Hill, NJ 07974, USA \\ 3 Departmento de Astronomia, Universidad Católica, Santiago, Chile \\ 4 Dept. of Astron. and Physics, University of Washington, Seattle, WA 98195, USA \\ 5 AURA Observatory, Calle Mariategui 2438, Providencia Santiago, Chile \\ 6 Research School of Astron. and Astrophys., ANU, Canberra, ACT 2611, Australia \\ 7 Dept. of Phys. and Astron., McMaster University, Hamilton, ON L8S 4M1, Canada
}

\begin{abstract}
Past microlensing experiments such as the MACHO project have discovered the presence of a larger than expected number of microlensing events towards the Large Magellanic Cloud (LMC). These events could represent a large fraction of the dark matter in the halo of our Galaxy, if they are indeed due to halo lenses. However, the locations of most of the lenses are poorly defined. The SuperMacho project will detect and follow up $\sim 60$ microlensing events toward the LMC over the next 5 years. The expected discovery of a number of microlensing events exhibiting special properties due to binarity, etc., will allow us to better determine the location and nature of the lenses causing the LMC microlensing events.
\end{abstract}

\section{Introduction}

The MACHO collaboration observed 17 microlensing events towards the LMC over 5.7 years [6]. These events point to a MACHO dark halo fraction of $8-50 \%$ at a $95 \%$ confidence level for a typical halo model with a peak likelihood of $20 \%$. However, this result depends on the location of the events and our knowledge of the shape and the extent of the dark halo. The current large uncertainties are primarily due to the small number of observed events. To reduce uncertainties requires a large number of microlensing events.

The SuperMacho project has observed the LMC for $\frac{1}{2}$ a night every second night within ten days of new moon from November 2001 to January 2002. The project has been allocated time to continue this work for 4 more years. Followup of microlensing events has not yet commenced but will be carried out at the Boyden 60-inch telescope in South Africa and at the 50-inch MACHO telescope in Australia.

\subsection{The Microlenses}

The measured $20 \%$ MACHO halo fraction significantly exceeds the known components due to foreground populations and self-lensing (a few events expected 
compared to the 17 observed [12]). The exact nature of the lensing objects remains a mystery.

The possible sources of lenses include:

- Ordinary stars in a previously undetected thick disk component.

- LMC stars as sources and lenses (so called self-lensing).

- An intervening dwarf galaxy or an unvirialized shroud component.

- Dark matter in the form of MACHOs.

Possible sources of ordinary stars producing the events include the halo white dwarfs which are currently being found in proper motion surveys [15] \& [14]. However, these results are controversial [17]. The self-lensing explanation is unlikely due to LMC's small observed scale height [16]. The existence of a foreground intervening population is strongly constrained by past observations [10] $\&$ [11], as is a virialized halo. However, the presence of a background population is less well constrained [8]. A dark matter solution remains likely.

Based on Holtzman et al. (1999)'s [13] HST LMC luminosity function, and an exponential disk of scale length $1.6 \mathrm{kpc}$, the SuperMacho project will monitor 500 million (100 million with $\mathrm{S} / \mathrm{N}>10)$ LMC stars compared to MACHO project's 11.9 million stars. This will allow SuperMacho to move from small number statistics (13-17 events [6]) to respectable numbers. We expect $>12$ events $\mathrm{yr}^{-1}$ or $\sim 60$ events overall.

\section{Microlensing Events}

The magnification of the source star during a gravitational microlensing event is related to the lens-source alignment impact parameter $u$ by,

$$
M(u)=\frac{u^{2}+2}{u\left(u^{2}+4\right)^{\frac{1}{2}}}
$$

In Fig. 1 we show the shapes of point-source, point-lens, microlensing light curves. The timescale of microlensing events, $\hat{t}$, is characterised by the time it takes for the Einstein ring associated with a foreground object to transit the background source star. The projected size of the Einstein ring radius, $R_{E}$, is given by

$$
R_{E}=\sqrt{\frac{4 G M D_{d}}{D_{s} c^{2}}\left(D_{s}-D_{d}\right)}
$$

where the lens has mass $M$ (in units of $\mathrm{M}_{\odot}$ ), the observer-lens distance is $D_{d}$ and source-observer distance is $D_{s}$. The timescale of lensing events is related to the projected transverse velocity of the lens $v_{\perp}$, by $\hat{t} \equiv 2 R_{E} / v_{\perp}$. 


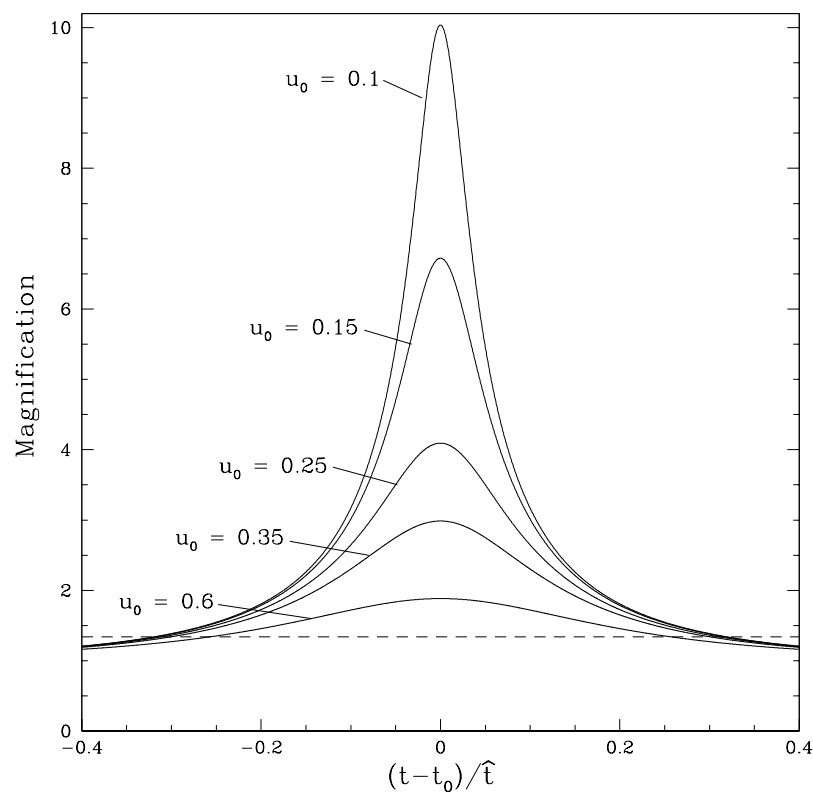

Fig. 1. The shapes of microlensing light curves for a variety of impact parameter values. The dashed line shows the magnification at one Einstein ring radius separation.

\subsection{Exotic Events}

During microlensing only the event magnification and timescale are observed. As we can see from equation (2), the timescale involves the mass, the distance and the projected transverse velocity of the lens. Thus, the dependence of $R_{E}$ on the location and lens mass makes it difficult to determine the physical parameters of individual microlensing events. For typical point-source, point-lens microlensing events it is not possible to separate these quantities. However, under special circumstances it is possible to break the degeneracy with exotic events. Instances of such exotic microlensing include:

- Events perturbed by the parallax effect [2]. In this case the orbital motion of the earth changes the observed line-of-sight and transverse velocity of the system. This distorts the light curve shape and allows us to constrain the transverse velocity of the source-lens pair.

- Events exhibiting the xallarap effect [9]. This occurs when the source star is part of a multiple system. Here the orbital motion of the sources perturb the observed light curve (xallarap is parallax backwards).

- Events showing finite source effects [3]. These effects occur when the impact parameter is very small (high magnification events). In such cases the pointsource, point-lens, approximation is violated and part of the source star may 
be resolved by the lens. These effects constrain the physical size of the event impact parameter.

- Events with binary lenses [19] \& [7]. In these cases the lenses can produce dramatic increases in magnification when caustics are crossed or a cusp is passed. For the binary event 98 -SMC-1, the MACHO group was able to show the location of the lens was within the SMC [5] based on the caustic crossing time, a velocity model, and the light curve shape.

Based on the number of microlensing exotic events observed toward the Galactic bulge ( $>20$ exotic events observed), we expect $\sim 10 \%$ of the SuperMacho events to provide additional information about physical parameters of the microlensing events observed by SuperMacho.

\section{Observations}

The observations for the SuperMacho project are underway at the Blanco 4$\mathrm{m}$ telescope at CTIO, Chile. During these observations 52 primary fields are monitored each night (23 square degrees) with an additional sixteen secondary fields monitored when time allows. Images are taken with the MosaicII imager. This is a prime focus imager with $8 \mathrm{CCDs}$ in $8 \mathrm{k} \times 8 \mathrm{k}$ mosaic $\left(0.27^{\prime \prime}\right.$ pixels $)$. A custom built wide-bandpass filter is employed for maximum photon throughput with minimum sky $\mathrm{OH}$ - and scattered moonlight. Because of the wide-bandpass atmospheric dispersion is an important factor in image quality. This light refraction is corrected on the telescope via an atmospheric dispersion corrector.

At CTIO the seeing is typically $\sim 0.8^{\prime \prime}$. The MACHO project median seeing was $\sim 2.1^{\prime \prime}$. With good seeing, a large telescope, sensitive CCDs and a wide bandpass filter, observations reach 2 magnitudes deeper than those in the MACHO experiment. A 120 second exposure reaches 23rd magnitude. However, exposure times of individual fields are optimized to produce the largest amount of stellar coverage in a given observing time. In Fig. 2 we present images of the same area of sky taken with the MACHO and SuperMacho telescopes. The superior seeing and depth of the CTIO observation are clear.

\section{Event Detection}

The image reduction and event detection is being carried out using Difference Image Analysis (DIA) [1] \& [4]. This technique produces better quality photometry in crowded fields than traditional PSF photometry and reduces the number of objects to the small number of variable sources. In the DIA scheme all images of a field are registered to a good seeing template image. Next the seeing and sky conditions in the images are matched by convolving the better seeing template image with the kernel required to match it to the poorer seeing images. The matched template image is then subtracted from the other images. What remains is a residual flux image (or difference image) containing only noise and point sources where the flux has varied between the image epochs. 

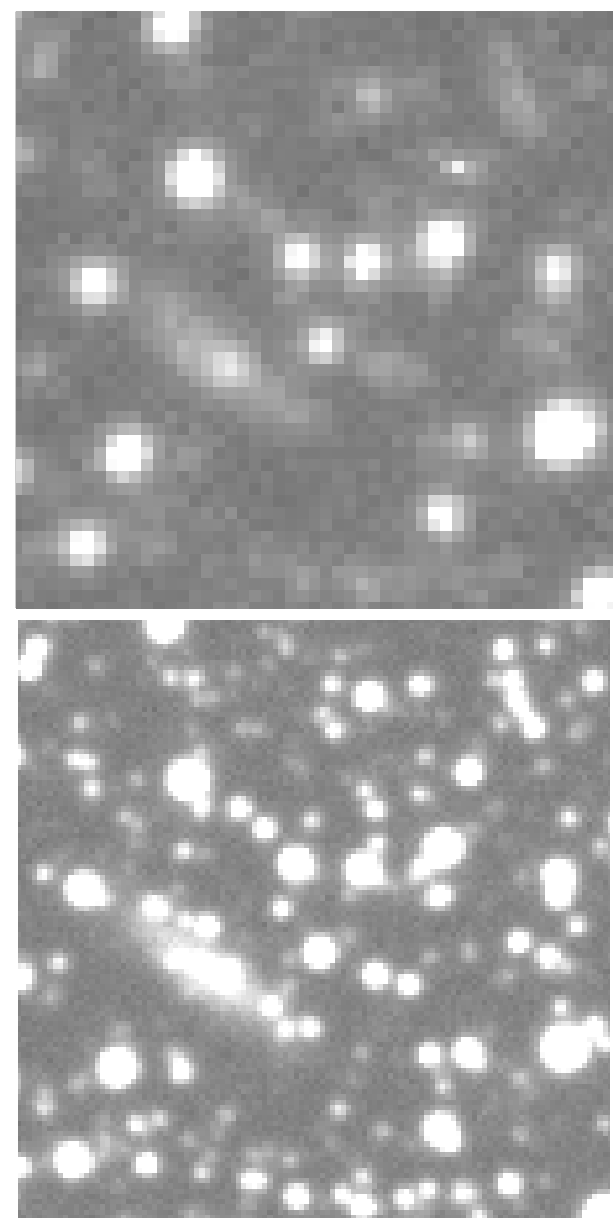

Fig. 2. A comparison of MACHO and SuperMacho images. Top: a typical MACHO image of a smaller region of the LMC. Bottom: the same region observed by the SuperMacho project.

These variable sources include microlensing events, variable stars, supernovae and asteroids. Once the full set of images has been differenced, photometry is performed on all the variable sources and light curves are produced. In Fig. 3 we present a template image and a difference image of the same region. The bright point sources in the difference image are objects that have become brighter in the time between observations, while the dark ones are those which have faded.

A microlensing event detection system is run over the light curves to find profiles which are consistent with the characteristics of microlensing events, for example see Fig. 1. To determine the optical depth to MACHO microlensing it is necessary to know the microlensing detection efficiency. This is determined by adding artificial stars of varying brightness to images during reduction. The 


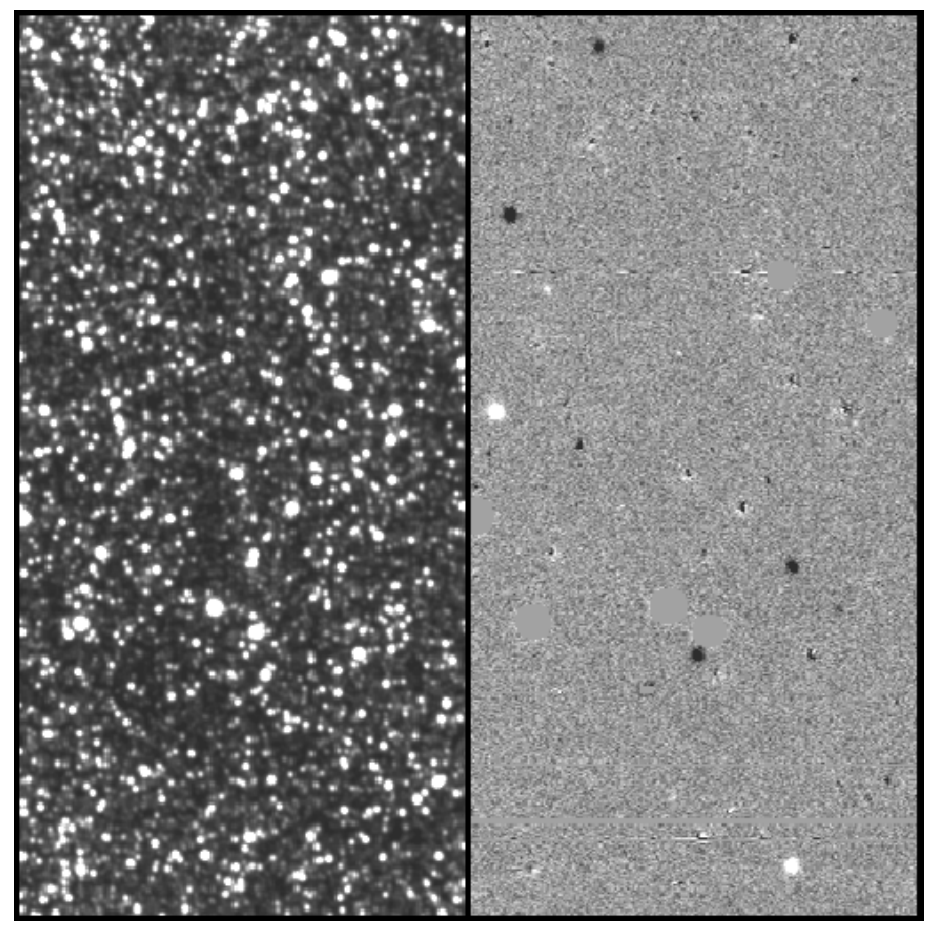

Fig. 3. A template image (left) and a difference image (right) of the same sky region.

fraction of recovered artificial stars gives the detection efficiency at a given magnitude limit. Reduced data, such as the photometry and locations of the sources, are stored in a "PostgreSQL" format database. This allows for quick searches of the indexed data and forward compatibility for inclusion of additional data fields. Each observing season will produce about $1 / 2$ a TByte of data to include in the database. The database of images is kept on disk for analysis of newly discovered sources at dates prior to their detection.

\section{Further Products of the Survey}

Besides microlensing events this new survey is providing many other data products. These include an accurate astrometric catalog of the LMC and deeper photometry of a large area of the LMC. An astrometric catalog will be useful for identifying optical sources in future surveys such as for X-ray sources. Amongst other things, the deep photometry will allow us to detect many $\delta$-Scuti type variables stars in the LMC. The survey may also discover near-earth and trans-Neptunian objects in unusual orbits far from the ecliptic. 
The MACHO project discovered a number supernovae behind the LMC. The increased depth of the SuperMacho data will allow us to detect many more supernovae. These data will supplement the small number of low redshift supernovae discovered so far by dedicated surveys. The first supernova of the project has already been discovered [18].

More than 50 QSOs have been detected behind the LMC by the MACHO project. The SuperMacho data will increase this by an order of magnitude. Such distant point sources as QSOs are necessary to accurately determine the proper motion of the LMC. This measurement will help us further constrain the mass and shape of the halo. Real time reduction will give other researchers the ability to follow up microlensing events and other transient sources. Studies at multiple wavelengths can also be undertaken simultaneously with these data for detailed studies of variability.

\section{Summary}

LMC microlensing events represent a large amount of hidden mass if the lenses reside within the dark halo of our galaxy. However, other possibilities exist and must be ruled out before conclusions can be made. SuperMacho will find and follow up $\sim 60$ microlensing events. These results, combined with those of the MACHO project, will provide a stronger constraint on the nature of LMC microlensing and our Galaxy's dark halo MACHO mass fraction. However, since only the halo microlensing events are relevant to the dark matter problem it is necessary to determine the locations of the lenses causing the observed events. Most of the lenses in microlensing events have unknown locations because of the well-known mass, distance, transverse velocity degeneracy. By using exotic microlensing events with well sampled light curves, it is possible to constrain the locations of the lenses. Based on previous microlensing experiments we expect to detect $>6$ exotic lensing events. By determining the locations of even a few of the lenses it is possible to solve the question of where most of the lenses towards the LMC reside.

\section{Acknowledgments}

We are grateful to the technical staff at the support staff at CTIO Observatories for their skilled support of the project. This work was performed under the auspices of the U.S. Department of Energy, National Nuclear Security Administration by the University of California, Lawrence Livermore National Laboratory under contract No. W-7405-Eng-48.

\section{References}

1. C. Alard, R. H. Lupton, Astrophys. Journal, 1998, 503, 325

2. C. Alcock, R.A. Allsman, et al., Astrophys. Journal, 1995, 454, 125 
A. J. Drake et al.

3. C. Alcock, R.A. Allsman, et al., Astrophys. Journal, 1997, 491, 436

4. C. Alcock, R.A. Allsman, et al., Astrophys. Journal, 1999, 521, 602

5. C. Alcock, R.A. Allsman, et al., Astrophys. Journal, 1999, 518, 44

6. C. Alcock, R.A. Allsman, et al., Astrophys. Journal, 2000, 542, 281

7. C. Alcock, R.A. Allsman, et al., Astrophys. Journal, 2000, 541, 270

8. C. Alcock, R.A. Allsman, et al., Astrophys. Journal, 2001, 552, 582

9. C. Alcock, R.A. Allsman, et al., Astrophys. Journal, 2001, 552, 259

10. A. Gould, Astrophys. Journal, 1998, 499, 728

11. A. Gould, Astrophys. Journal, 1999, 525, 734

12. G. Gyuk, N. Dalal, K. Griest, Astrophys. Journal, 535, 90

13. J. Holtzman, J.S. Gallagher, et al. 1999, Astronom. Journal, 118, 2262

14. C. Nelson, K. Cook, et al., Astrophys. Journal, 2002, in press

15. B.R. Oppenheimer, N.C. Hambley, et al., Science, 2001, 292, 698

16. P. Padoan, S. Kim, et al., Astrophys. Journal, 2001, 555, 33

17. N.I. Reid, K. Sahu, S.L. Hawley, Astrophys. Journal, 2001, 559, 942

18. C. Stubbs, A. Rest, et al., IAU Circular, 'Supernova 2002B', 2002, 7791,

19. A. Udalski, M. Szymański, et al., Astrophys. Journal, 1994, 436, 103 